\title{
Percepção dos profissionais quanto à pertinência dos indicadores de saúde bucal em uma metrópole do Nordeste brasileiro
}

\author{
Perception by professionals on the relevance of the oral health \\ indicators in a metropolis of the Brazilian northeastern
}

Francisco Ivan Rodrigues Mendes Júnior ${ }^{\mathbf{1}}$, Mary Anne Medeiros Bandeira², Fábio Solon Tajra ${ }^{\mathbf{3}}$

\begin{abstract}
RESUMO Estudo exploratório-descritivo, com abordagem quali-quantitativa, que buscou analisar a percepção dos profissionais quanto à pertinência dos indicadores de saúde bucal do Programa Nacional de Melhoria do Acesso e da Qualidade da Atenção Básica (PMAQ-AB) em Fortaleza, Ceará. A desmotivação, a descontinuidade no fornecimento de insumos odontológicos, o abandono do tratamento por parte do paciente, a cultura popular de que a gestante não pode realizar tratamento odontológico e a insegurança do profissional no manejo clínico relacionado à prótese dentária, ao atendimento à gestante, ao atendimento à urgência odontológica e ao diagnóstico de alteração da mucosa oral dos usuários foram problemas identificados na operacionalização desses indicadores e mencionados como interferência para o cumprimento de suas metas.
\end{abstract}

PALAVRAS-CHAVE Saúde bucal; Estratégia Saúde da Família; Avaliação em saúde.

ABSTRACT Exploratory-descriptive study, with qualitative and quantitative approach, which aims to analyze the perception by professionals on the relevance of oral health indicators of the National Program for Access and Quality Improvement of Primary Care (PMAQ-AB) in Fortaleza, Ceará. The demotivation, the discontinuity in providing dental inputs, the abandonment of treatment by the patient, the popular culture in which the pregnant cannot carry out the dental treatment and the insecurity of the professional in the clinical handling related to dental prosthesis, to the attendance of the pregnant, to the attendance of dental urgency and to the diagnosis of user's oral mucosa alteration were problems identified in the operationalization of these indicators and mentioned as interference for the fulfillment of their goals.

KEYWORDS Oral health; Family Health Strategy; Health evaluation.

1 Universidade Federal do Ceará (UFC) - Fortaleza (CE), Brasil.

ivanjrdentista@gmail.com

2 Universidade Federal do Ceará (UFC) - Fortaleza (CE), Brasil.

mambandeira@yahoo.

com.br

3 Universidade Federal do Piauí (UFPI) - Teresina (PI), Brasil.

fstajra@hotmail.com 


\section{Introdução}

Com a evolução das políticas públicas de saúde bucal no Brasil, muitos desafios foram manifestados. Isso pode ser percebido tanto na necessidade de demonstrar resolutividade na assistência clínica como na execução de políticas intersetoriais que possam influenciar positivamente o desenvolvimento dos principais indicadores epidemiológicos de saúde bucal (MOYSES, 2008).

Aliado a isso, há um esforço do Ministério da Saúde pela indução de uma gestão pública baseada no monitoramento e na avaliação de processos e resultados (BRASIL, 2012). A utilização desse padrão de avaliação passou a se constituir numa ferramenta importante para a qualificação das ações e do cuidado à saúde dos indivíduos, da família e da comunidade (ALMEIDA; GIOVANELLA, 2008).

Desse modo, muitas ações têm sido desenvolvidas no sentido de ajustar as estratégias previstas na Política Nacional de Atenção Básica (PNAB), com a intenção de reconhecer a qualidade dos serviços ofertados à sociedade brasileira. Para isso, o Ministério da Saúde implantou, em 2011, por meio da Portaria n. ${ }^{\circ} 1.654$ GM/MS, o Programa Nacional de Melhoria do Acesso e da Qualidade da Atenção Básica (PMAQ-AB), em que foram selecionados 47 indicadores, subdivididos em sete áreas estratégicas e classificados segundo a natureza de seu uso (BRASIL, 2012):

- Indicadores de desempenho: vinculados ao processo de avaliação externa e que serão utilizados para a classificação das equipes da Atenção Básica (AB), conforme o seu desempenho;

- Indicadores de monitoramento: a serem acompanhados de forma regular para complementação de informações sobre a oferta de serviços e resultados alcançados por equipe, sem, no entanto, influenciar a pontuação atribuída às equipes no processo de avaliação externa.
Diante de uma série de indicadores de desempenho e monitoramento, apontados e categorizados por meio desse programa, surgiram alguns questionamentos: qual é a percepção dos profissionais quanto à pertinência dos indicadores de saúde bucal do PMAQ-AB? Quais seriam as barreiras para o seu monitoramento?

Neste sentido, esta pesquisa visou a analisar a percepção dos profissionais quanto à pertinência dos indicadores em saúde bucal do Programa Nacional de Melhoria do Acesso e da Qualidade da Atenção Básica em Fortaleza, Ceará.

Espera-se que os resultados obtidos nesta pesquisa possam provocar reflexões dos sujeitos envolvidos nesses processos, de tal forma que tragam possíveis ajustes nas melhorias da qualidade da atenção à saúde bucal.

\section{Métodos}

Trata-se de um estudo exploratório-descritivo, com abordagem quali-quantitativa, realizado no período de outubro de 2013 a março de 2014, que contou com uma análise documental prévia para caracterização do cenário e do perfil dos profissionais envolvidos, seguido da aplicação de um questionário semiestruturado, em que se pôde captar a percepção do grupo de CirurgiõesDentistas (CDs) frente à operacionalização dos indicadores de saúde bucal do PMAQ-AB e à sua atuação na Estratégia Saúde da Família (ESF).

Para que isso fosse efetivado, contou-se com a apreciação do Comitê de Ética em Pesquisa da Universidade Federal do Ceará (UFC), Parecer n. ${ }^{\circ}$ 443.474, sendo seguidas as normas da Resolução CNS n466/12.

A coleta de dados secundários se deu por meio da obtenção de informações acerca do cadastro dos CDs da ESF no Sistema de Cadastro Nacional de Estabelecimento de Saúde (SCNES), da população geral do município de Fortaleza, Ceará, no Instituto 
Brasileiro de Geografia e Estatística (IBGE) e no Departamento de Informática do SUS (Datasus).

Para a coleta de dados primários, foi utilizado um questionário contendo três partes, a saber: perfil dos CDs da ESF; percepção acerca dos indicadores de desempenho; e percepção acerca dos indicadores de monitoramento (ver quadro 1). O questionário era composto por 37 perguntas, sendo 23 questões fechadas e 14 questões abertas.

Quadro 1. Registro de indicadores de saúde bucal do Programa Nacional de Melhoria do Acesso e da Qualidade da Atenção Básica (PMAQ-AB/saúde bucal)

Indicadores de Desempenho

Média da ação coletiva de escovação dental supervisionada;

Cobertura de primeira consulta odontológica programática;

Razão entre tratamentos concluídos e primeiras consultas odontológicas;

Proporção de instalações de próteses dentárias.

Indicadores de Monitoramento

Cobertura de atendimento odontológico à gestante;

Média de atendimentos de urgência odontológica por habitante;

Taxa de incidência de alterações na mucosa oral.

Fonte: BRASIL, 2012

Inicialmente, foi aplicado um pré-teste, com seis CDs que tinham atuado como Coordenadores Regionais de Saúde Bucal, no município de Fortaleza, no ano de 2012. Nesse momento, cada CD pôde solicitar ao pesquisador os esclarecimentos necessários acerca de possíveis dúvidas na resolução do questionário, assim como sugerir possíveis modificações no instrumento a ser utilizado. Dessa forma, foi possível realizar os ajustes necessários para uma melhor compreensão das questões contidas no instrumento.

No que diz respeito à cobertura populacional da ESF, o município de Fortaleza passou por uma evolução relevante. De uma cobertura populacional de $11,77 \%$, em 2005 , passou para $36,84 \%$, em outubro de 2013. Nessa ocasião, encontravam-se implantadas 267 Equipes de Saúde da Família (EqSF) e 195 Equipes de Saúde Bucal (EqSB). Foi diante desse cenário que se desenvolveu a presente pesquisa.

Após prévia articulação junto à Coordenação Municipal de Saúde Bucal (CMSB), foram identificados todos os profissionais a serem pesquisados e programada a aplicação dos questionários nos encontros de Educação Permanente (EP), promovidos pela gestão municipal. Com relação aos CDs que não participaram do processo de EP, foram feitas buscas ativas por meio dos coordenadores de saúde bucal das seis regionais de saúde.

Diante dos profissionais, foram feitas as considerações acerca da importância da pesquisa a ser realizada, bem como dos seus principais objetivos. Logo após, os questionários foram aplicados aos pesquisados que se dispuseram a assinar o Termo de Consentimento Livre e Esclarecido (TCLE).

Para digitação do banco de dados, utilizou-se o software Microsoft Excel, e, para sua consolidação e análise estatística, o software Statistical Package for Social Science for Windows (SPSS), de tal forma que se permitiu visualizar os resultados de forma simples e autoexplicativa, possibilitando, assim, associar pesquisas de opinião, atitudes, pontos de vista e preferências que os pesquisados e autores com expertises na área têm a respeito dos temas em questão. 


\section{Resultados e discussão}

Os resultados a serem apresentados são derivados dos questionários aplicados aos 137 CDs da ESF do município de Fortaleza, inseridos nas seis regionais de saúde. Esse quantitativo de CDs correspondeu a 70,25\% de um universo de 195 CDs cadastrados nas EqSB em outubro de 2013.
Cerca de $76 \%(n=104)$ dos pesquisados corresponderam ao sexo feminino. Além disso, 65\% ( $\mathrm{n}=89)$ dos CDs estavam com idade acima de 35 anos, e $58,4 \%(n=80)$ apresentaram mais de 6 anos de tempo de serviço na ESF (tabela 1). A partir desses dados, foi possível estimar que esses profissionais são, de certa forma, experientes no serviço.

Tabela 1. Distribuição dos 137 Cirurgiões-Dentistas da Atenção Básica do município segundo características pessoais e formação acadêmica, Fortaleza, Ceará, 2014

\begin{tabular}{|c|c|c|}
\hline VARIÁVEIS & $\mathbf{N}$ & $\%$ \\
\hline \multicolumn{3}{|l|}{ Sexo } \\
\hline Feminino & 104 & 75,9 \\
\hline Masculino & 31 & 22,6 \\
\hline Não informou & 2 & 1,5 \\
\hline \multicolumn{3}{|l|}{ Idade } \\
\hline Entre 25 e 35 anos & 48 & 35,0 \\
\hline Acima de 35 anos & 89 & 65,0 \\
\hline \multicolumn{3}{|l|}{ Tempo de serviço } \\
\hline$<1$ ano & 2 & 1,5 \\
\hline 1 a 3 anos & 40 & 29,2 \\
\hline 3 a 6 anos & 13 & 9,5 \\
\hline$>6$ anos & 80 & 58,4 \\
\hline Não informou & 2 & 1,5 \\
\hline \multicolumn{3}{|l|}{ Outro tipo de ocupação } \\
\hline Sim & 76 & 55,5 \\
\hline Não & 60 & 43,8 \\
\hline Não informou & 1 & 0,7 \\
\hline \multicolumn{3}{|l|}{ Tipo de ocupação (n=76) } \\
\hline Consultório Particular & 57 & 75,0 \\
\hline Outro & 19 & 25,0 \\
\hline \multicolumn{3}{|l|}{ Tipo de IES em que se formou } \\
\hline Pública & 98 & 71,5 \\
\hline Privada ou Filantrópica & 39 & 28,5 \\
\hline Realizou pós-graduação anterior & 128 & 93,4 \\
\hline Aperfeiçoamento & 77 & 56,2 \\
\hline Especialização & 121 & 88,3 \\
\hline Mestrado & 18 & 13,1 \\
\hline Doutorado & 2 & 1,5 \\
\hline Pós-graduação em andamento & 33 & 24,1 \\
\hline Aperfeiçoamento & 4 & 2,9 \\
\hline Especialização & 16 & 11,7 \\
\hline Mestrado & 8 & 5,8 \\
\hline Doutorado & 5 & 3,6 \\
\hline
\end{tabular}

Fonte: Elaboração própria 
Em torno de $75 \%(n=57)$ dos respondentes relataram exercerem essa ocupação em consultórios particulares. Diante desse quadro, vale acrescentar que:

se observa a existência de profissionais com vínculos públicos parciais, como os que exercem a profissão em serviços públicos e possuem consultórios privados como autônomos. Ainda há os que possuem consultório, não possuem vínculo empregatício com os serviços públicos, mas são credenciados pelo SUS. Outros são assalariados de empresas privadas de serviços odontológicos, empresas que oferecem serviços de odontologia aos seus funcionários, sindicatos e também há os que se organizam em grupos de cooperativas. (MORITA; HADDAD; ARAÚJO; 2010, P. 68).

Outro dado importante a ser considerado é que $93,4 \%$ ( $n=128)$ dos CDs pesquisados informaram já terem realizado pós-graduação, seja na condição de aperfeiçoamento $(56,2 \%)$, especialização (88,2\%), mestrado (13,1\%) ou doutorado (1,5\%). Acrescenta-se a esses dados o quantitativo de $24,1 \%$ ( $n=33$ ) de CDs que se encontram em pós-graduação, seja em aperfeiçoamento (2,9\%), especialização (11,7\%), mestrado (5,8\%) ou doutorado (3,6\%).

Dos 137 CDs pesquisados, 97,1\% (n=133) já realizaram ou realizam alguma pós-graduação. Destes, 59,8\% ( $\mathrm{n}=82)$ estão associados com pós-graduação nas áreas de saúde da família, saúde pública e/ou saúde coletiva.

Para Freitas (2007, P.26),

- mercado de trabalho odontológico passa a sofrer modificações mais acentuadas a partir da década de 1980. Vários estudos sinalizam para a tendência do assalariamento, do trabaIho no consultório com convênios e credenciamentos e a associação de ambas as formas.

Neste sentido, mesmo com 41,6\% dos CDs da ESF exercendo ocupações em consultórios particulares, parece ter havido o despertar do interesse dos CDs para realização de cursos de pós-graduação nas áreas de saúde da família, saúde pública e/ou saúde coletiva, possivelmente em virtude das necessidades sentidas por eles de desenvolvimento do seu trabalho nas equipes da ESF, assim como pela possibilidade de ascensão salarial do Plano de Cargos, Carreiras e Salários (PCCS) da Prefeitura Municipal de Fortaleza.

A segunda parte do questionário correspondeu à análise de pertinência dos indicadores de desempenho de saúde bucal. Para o indicador de desempenho da Média de Ação Coletiva de Escovação Dental Supervisionada (Maceds), com exceção de um CD, todos os outros consideraram esse indicador importante, e $94,9 \%(n=130)$ deles responderam sentirem-se capacitados para essa ação.

Quanto à classificação do grau de importância da Maceds, 75,9\% ( $\mathrm{n}=104)$ dos CDs responderam serem as creches e escolas o principal espaço para realização dessa ação. 64,9\% (n=89) dos CDs elegeram, em segundo lugar, os domicílios como espaço para realização dessas atividades, enquanto $51,8 \%(\mathrm{n}=71)$ dos entrevistados responderam serem as Unidades Básicas de Saúde (UBS) o local preferido. Já 49,6\% (n=68) responderam serem outros espaços importantes para o desenvolvimento dessas ações, tais como: espaços de convivência para idosos, espaços de reabilitação para adolescentes e outros.

Para Waat (2007), a promoção de saúde bucal deve ser garantida por meio da implementação de estratégias múltiplas em ambientes diferentes, como domicílios, escolas, creches, centros comunitários, locais de trabalho. Dessa forma, quanto mais espaços forem identificados para oportunizar esse tipo de prática, maior será a possibilidade de sucesso e mais efetiva será sua ação.

Contudo, mesmo a ideia de promoção da saúde em escolas não sendo nova (BEATTIE, 1996), os resultados provenientes da Maceds demostram ter efeitos mínimos e de curta duração, principalmente pela forma como é desenvolvida (NUTBEAM, 1997). Nesse sentido, devem ser somados esforços e aglutinados recursos que 
oportunizem a melhoria das condições de saúde bucal da população. Cabe, ainda, questionar acerca das estratégias de continuidade da ação e da formação de multiplicadores que possam agregar maior impacto na saúde da comunidade assistida.

Para que as práticas de educação em saúde sejam mais sensíveis às necessidades subjetivas e culturais dos usuários, faz-se necessário abandonar estratégias comunicacionais informativas e adotar uma comunicação dialógica (ALVES, 2005).

A educação em saúde bucal voltada a escolares deve ser integrada à educação em saúde geral, e a higiene bucal deve ser integrada à educação em saúde, voltada aos cuidados e à higiene de todo o corpo, trabalhando com a autoestima da criança (SHEIHAM, 1983). No caso de Fortaleza, para 65,7\% ( $\mathrm{n}=88)$ dos CDs, a desmotivação é a principal dificuldade para o desenvolvimento dessa atividade. Em segundo lugar, 53,3\% (n=72) dos CDs destacaram a falta de planejamento e articulação intersetorial, seguidos pela descontinuidade no fornecimento de kits $(48,1 \%)$ e espaços sem estruturas físicas (36,3\%).

Ao se avaliar a percepção dos CDs sobre o indicador de desempenho de Cobertura de Primeira Consulta Odontológica Programática, tem-se que $91,2 \% \quad(n=125)$ consideraram esse indicador importante, enquanto que $5,8 \%(n=8)$ consideraram-no sem importância - e quatro não responderam.

Para os que consideraram o indicador importante, as principais justificativas foram: "Retrata a questão do acesso ao serviço e a continuidade do tratamento odontológico" (Informante-chave 18); "Porque assim podemos observar se a saúde bucal da população está melhorando ou não, bem como nos auxilia no planejamento do tratamento preventivo e curativo" (Informante-chave 87); "Porque sinaliza o acesso do paciente ( $1^{a}$ vez), onde ele tem a oportunidade de concluir o tratamento através da motivação do profissional, que pode ser descontinuada com a falta de material" (Informante-chave 49).
Já para os CDs que responderam não ter importância esse indicador, seguem algumas justificativas relatadas:

No momento, este indicador não está mostrando realmente a realidade, pois muitos alimentam este dado erroneamente, colocando como primeira consulta a ida do paciente ao consultório, sem odontograma e planejamento. (Informante-chave 47);

"Porque ela não condiz com a realidade do acesso" (Informante-chave 80); "Não é fiel" (Informante-chave 101).

Quanto ao indicador de Razão entre Tratamentos Concluídos e Primeiras Consultas Odontológicas Programáticas, 93,4\% ( $n=128)$ dos CDs consideram esse indicador importante. Isso pode ser observado em alguns relatos com a seguinte justificativa: "Porque permite avaliar a resolutividade dos serviços" (Informante-chave 2); "Para quantificar o número de usuários que têm acesso e conseguem terminar o tratamento" (Informante-chave 12); "Porque podemos avaliar se há necessidade ou não de ajustes no processo de trabalho" (Informante-chave 87).

Para os CDs que não consideraram importante esse indicador, tivemos como justificativa: "Não há, ainda, o tratamento iniciado e acabado, pois tem demanda livre, urgência, e o próprio usuário não tem uma mentalidade responsável para encarar o tratamento" (Informante-chave 5); "Não refletem a realidade. Não há conexão entre o problema e a prática. Não há ciência" (Informante-chave 8); "É um indicador fora da realidade, pois há muitas falhas nas informações" (Informante-chave 118).

Apesar de a grande maioria dos CDs considerar esse indicador importante, os relatos demonstraram que também o considera complexo, em especial, nos quesitos associados aos registros dos tratamentos concluídos e às primeiras consultas odontológicas programáticas. Ou seja, consideram a concepção do indicador importante, 
porém, têm consideráveis dificuldades em sua construção.

Outra questão abordada junto aos CDs foi o grau de dificuldade apresentado por eles na execução de procedimentos clínicos em saúde bucal e na conclusão de tratamentos odontológicos. Para 48,5\% ( $\mathrm{n}=65)$ dos CDs, a maior dificuldade apresentada para execução dos procedimentos clínicos é a descontinuidade no fornecimento de insumos odontológicos, seguida por outras causas, tais como: "Auxiliar em saúde bucal com treinamento deficiente" (Informantechave 5); "Falta de Técnico em saúde bucal nas equipes, que poderiam ser utilizadas em ações delegáveis a estes profissionais” (Informante-chave 37); "Deveria ter capacitação para dentistas solucionarem situações específicas" (Informante-chave 51); "Muitas faltas dos pacientes, bem como abandono do tratamento, problemas socioeconômicos e dificuldade de motivação" (Informante-chave 105); "Poucos consultórios e baixa cobertura de Equipe de Saúde Bucal no território" (Informante-chave 114).

Como terceira maior dificuldade, 44,7\% $(n=59)$ dos CDs consideraram ser o elevado número de pacientes com necessidades para atividades clínicas. A deficiência estrutural (falta de insumos, equipamentos danificados e pouca manutenção) foi considerada a quarta maior dificuldade para execução das ações clínicas.

Com relação às dificuldades encontradas para conclusão dos tratamentos odontológicos programáticos, 44,3\% $(n=58)$ dos CDs responderam como principal causa o abandono do tratamento por parte do paciente, enquanto que $43,5 \%(n=57)$ associaram essa dificuldade à deficiência estrutural (falta de insumos, equipamentos danificados e pouca manutenção). Outras dificuldades apontadas por alguns dos dentistas foram: "Falta espaço na agenda para marcar retorno toda semana ou quinzenal" (Informante-chave 53); "Falta de Equipe de Saúde Bucal nos CSF. Áreas descobertas”
(Informante-chave 93); "A falta do paciente ao trabalho, pois muitas empresas e patrões não aceitam a declaração de comparecimento como justificativa de falta ao trabalho" (Informante-chave 24); "O paciente do SUS não tem a mentalidade do paciente que paga um tratamento. São propensos a resolver só $o$ que lhes incomoda" (Informante-chave 5); "Dificuldade de recursos humanos e disponibilidade de horários na coordenação odontológica (divisão de horários com outros dentistas)" (Informante-chave 112).

Em último, entre as alternativas apresentadas, obteve-se que $34,9 \%(n=45)$ dos CDs responderam ser o elevado número de pacientes com necessidades para as atividades clínicas (grande demanda) também um fator que dificulta a conclusão dos tratamentos.

Ao se analisar o indicador de Proporção de Instalação de Próteses Dentárias na ESF, $75,2 \%(n=103)$ dos CDs responderam ser importante a instalação de próteses dentárias na ESF, enquanto que $23,4 \%(n=32)$ responderam que não, conforme se constata nas falas abaixo: "As próteses devem ser confeccionadas por especialistas na área" (Informantechave 43); "Enquanto a população apresentar outros graves problemas bucais, prótese é mais indicada para o Centro de Especialidades Odontológicas" (Informante-chave 49); "Não sabemos como realizar a instalação. Esse é um procedimento especializado" (Informantechave 80); "Acredito que deve ser realizado pelo profissional especialista e não pelo generalista" (Informante-chave 114).

A instalação de próteses dentárias na ESF não é assunto recente. Desde a sua incorporação como procedimento básico, tem gerado grande discussão. No que diz respeito ao benefício para a qualidade de vida dos pacientes, não há divergência. Hamalainen et al. (2005) mencionam que a perda dos dentes no idoso pode ser considerada um fator preditivo significativo para um maior nível de mortalidade, em virtude do comprometimento da qualidade da alimentação. Contudo, a possibilidade de realização desse 
procedimento na ESF não tem sido garantida por todos os profissionais.

Buscou-se identificar, ainda, as principais dificuldades encontradas pelos CDs para instalação de próteses dentárias na ESF. Para $50,4 \%(n=64)$ dos CDs, a principal dificuldade encontrada é a insegurança do profissional no manejo clínico. Já 44,1\% $(n=56)$ dos CDs declararam como segunda maior causa de dificuldade: "Falta atualização/capacitação em prótese para os dentistas e auxiliares. Há formação deficiente em prótese na graduação" (Informante-chave 14); "Falta de um bom laboratório de prótese" (Informante-chave 122); "Laboratório demora a entregar o trabalho" (Informantechave 58); "Demora na fila do laboratório" (Informante-chave 105).

A deficiência estrutural (falta de insumos, equipamentos danificados e pouco instrumentais) foi apresentada por $42,3 \%(n=55)$ dos CDs como sendo a terceira principal dificuldade. A quarta maior dificuldade, relatada por $36,2 \%(n=47)$ dos pesquisados, foi o elevado número de pacientes com necessidades para as atividades clínicas.

É importante mencionar que a reposição de dentes perdidos com a oferta de prótese na ESF, a melhora do acesso aos serviços e o funcionamento das unidades de saúde em horários alternativos poderiam reduzir, de certa forma, algumas iniquidades sociais em saúde bucal (MIOTTO ET AL., 2012).

A terceira parte do questionário correspondeu à avaliação das necessidades identificadas pelos CDs da ESF para o cumprimento dos indicadores de monitoramento em saúde bucal no município de Fortaleza, Ceará.

Para o indicador de monitoramento acerca da Cobertura de Atendimento Odontológico à Gestante, 89,1\% $(n=122)$ dos CDs consideraram esse indicador importante, no entanto, $8 \%(n=11)$ relataram não ser importante, conforme se observa nos relatos abaixo: "Acho que esse é um público bastante restrito quando comparado à demanda total da população" (Informante-chave
15); "Entendo que este indicador serve para comparar o número de gestantes cadastradas e acompanhadas pela PSF e o número de gestantes com acompanhamentos odontológicos, mas acredito que esse indicador possa gerar distorções...” (Informante-chave 42); "Teoricamente, todos deveriam ter o acesso garantido" (Informante-chave 101); "A maioria só comparece à $1^{a}$ consulta e não retorna para dar continuidade ao tratamento" (Informante-chave 110); "O importante é atender" (Informante-chave 118).

Chiaratto, Brandão e Souza (2003) consideram o período gestacional a fase em que as mulheres se mostram mais receptivas a informações relacionadas aos seus cuidados e ao futuro bebê. Isso demonstra a importância de desenvolver ações educativas em saúde nesse período da vida das mulheres.

Quando questionados se se consideravam capacitados para o atendimento à gestante, $86,1 \%(n=118)$ responderam que sim, enquanto 10,2\% (n=14) responderam que não, e cinco não responderam. Esse quantitativo percentual que não se considera capacitado sugere uma fragilidade na formação e no desenvolvimento profissional, tanto em meio acadêmico como na possibilidade de contato interdisciplinar em exercício na ESF. Mais uma vez, parece ser oportuna a implementação de políticas públicas de EP, em que possam, gradativamente, tornar os CDs mais seguros na execução desses procedimentos clínicos tão importantes para a saúde da gestante e, até mesmo, para o futuro bebê.

Ao se questionar a respeito do grau de dificuldade encontrado pelos CDs para o atendimento odontológico programático à gestante, obteve-se que a principal dificuldade para 39,5\% $(n=49)$ está relacionada com a cultura popular de que a gestante não pode realizar tratamento odontológico. Em segundo lugar, para 39,3\% ( $n=48)$, a insegurança no manejo clínico junto às gestantes foi o fator fundamental. E, por fim, 33,9\% $(n=42)$ relataram serem poucos os encaminhamentos 
das gestantes feitos pelos profissionais, em especial, médicos e enfermeiros, para a saúde bucal. Assim como elencaram outros motivos, tais como: "Falta de interesse da gestante" (Informante-chave 1); "Falta de insumos e atendentes. Equipamentos danificados, que impedem a conclusão do tratamento da gestante durante o periodo gestacional" (Informante-chave 14); "Falta de informação à gestante da importância de ter uma saúde bucal boa" (Informante-chave 19); "Stress da gestante, enjoo e dificuldade de caminhar até o ponto quando em estado avançado de gravidez" (Informante-chave 24); "Falta de tempo para atender à demanda" (Informantechave 39).

A baixa percepção das próprias necessidades odontológicas e o medo de se submeter à situação de tratamento são fatores que interferem na decisão da gestante pela busca de atenção odontológica (ALBUQUERQUE; ABEGG; RODRIGUeS, 2004). Para Maeda, Imparato e Bussadori (2005), a crença de que o tratamento odontológico conduzido na gestação poderia ser prejudicial à criança não é mantida só pelas gestantes, mas também por alguns profissionais de Odontologia, que, por falta de conhecimento, não se sentem seguros para tratar dessa clientela.

Para o indicador de monitoramento da Média de Atendimentos de Urgência Odontológica por Habitante, perguntou-se, inicialmente, se os CDs consideravam esse indicador importante. Para 89,8\% ( $\mathrm{n}=123)$ deles, a resposta foi sim, enquanto $8,0 \%$ $(\mathrm{n}=11)$ responderam não, conforme se constata nas falas descritas abaixo: "Atrapalha o objetivo do Programa Saúde da Família, devendo ser de responsabilidade do serviço próprio de urgência e emergência" (Informante-chave 24); "Não há tempo suficiente para o atendimento clínico" (Informante-chave 56); "Há falta (carência) de serviços especializados de urgência" (Informante-chave 81); "Não há organização do serviço para esse fim" (Informante-chave 80); "As Unidades de Pronto Atendimento deveriam assumir esse serviço" (Informante-chave 96).
No que diz respeito à avaliação do grau de dificuldade apresentado pelos CDs para $o$ atendimento das urgências odontológicas, $56,9 \%(\mathrm{n}=70)$ dos entrevistados citaram a insegurança do profissional CD no manejo clínico. Na sequência, foram mencionados o elevado número de pacientes com necessidades para as atividades clínicas (grande demanda) e a deficiência estrutural (falta de insumos, equipamentos danificados e pouco instrumental).

Ainda no tocante às principais dificuldades encontradas pelos CDs em seu manejo clínico para os atendimentos de urgências odontológicas, para 41,5\% $(\mathrm{n}=49)$ dos CDs, a principal dificuldade apresentada é a abertura/acesso coronário. Em segundo lugar, $\operatorname{com} 38,5 \%(n=45)$ do total dos entrevistados, ficou o diagnóstico diferencial, seguido da drenagem de abscesso e de outras causas, tais como: "Falta de $R X$ para diagnóstico diferencial" (Informante-chave 24); "Falta de insumos e estrutura física (equipamentos)" (Informante-chave 33); "Falta de valorização por parte da gestão em relação à odontologia” (Informante-chave 77); "Insegurança do profissional" (Informante-chave 89); "Falta de interesse por parte do profissional" (Informante-chave 99).

A última abordagem feita aos CDs dizia respeito à Taxa de Incidência de Alterações na Mucosa Oral de usuários atendidos pelas EqSB. Cerca de 92\% ( $n=126)$ dos entrevistados consideraram importante o diagnóstico de alteração da mucosa oral dos usuários, fato também considerado oportuno para que a gestão possa implementar políticas públicas que venham a promover o diagnóstico precoce de lesões pré-cancerígenas na Atenção Básica.

Quanto às principais dificuldades apresentadas pelos CDs para o diagnóstico de alteração da mucosa oral dos usuários, 47,5\% ( $\mathrm{n}=58)$ dos entrevistados citaram a insegurança do CD no manejo clínico. Em segundo lugar ficou a deficiência estrutural (falta de insumos, equipamentos danificados e poucos instrumentais), seguido pelo 
elevado número de pacientes com necessidades para as atividades clínicas (grande demanda), além de outras razões, tais como: "Estes pacientes inicialmente procuram o médico, que, geralmente, não nos encaminha tal paciente. Medicam erroneamente e retardam o diagnóstico. Dificuldade na referência para especialistas" (Informante-chave 5); "Não conhecimento do diagnóstico diferencial de várias lesões" (Informante-chave 47); "Não ter para onde encaminhar o paciente para um especialista realizar biópsia. Sem disponibilidade da estrutura secundária e terciária" (Informante-chave 96); "Falta de interesse por parte do profissional" (Informante-chave 99); "Falta de registro e encaminhamento para atenção secundária" (Informante-chave 114).

Para McDowel (2006), todos os pacientes devem ser examinados à presença de lesões pré-cancerosas e cancerosas, uma vez que o câncer oral pode ocorrer com ou sem a presença dos fatores de risco relacionados.

Para muitos autores, embora o diagnóstico dessas lesões seja acessível ao exame clínico, há evidências de que esse tipo de câncer ainda é diagnosticado muito tardiamente. Como consequência disso, observa-se, comumente, a necessidade de tratamento mutilador (GOMEZ ET AL., 2010).

Após análise dos dados obtidos dos questionários respondidos pelos CDs, identificaram-se algumas dificuldades na condução clínica de seus pacientes. Com isso, acredita-se que seja importante que a gestão municipal de saúde bucal promova, nos momentos dedicados ao processo de EP, oficinas de trabalho relacionadas aos cuidados clínicos em saúde bucal das gestantes, às urgências odontológicas, às confecções de próteses dentárias, ao diagnóstico de alteração da mucosa oral, assim como ao correto preenchimento de procedimentos nos sistemas de informação ambulatorial e da Atenção Básica.

Com relação ao local de registro dos procedimentos nos sistemas de informação vigentes, apontados pelos CDs, tivemos que, para o indicador de desempenho Maceds, apenas $26,3 \% \quad(n=36)$ dos entrevistados souberam informar o local correto para registro, conforme se observa na tabela 2 . Enquanto que, para os indicadores de desempenho de Cobertura de Primeira Consulta Odontológica Programática e de Proporção de Instalações de Próteses Dentárias, esses resultados foram de 40,9\% $(n=56)$ e $21,9 \%(n=30)$, respectivamente. Para os indicadores de monitoramento de Cobertura de Atendimento Odontológico à Gestante, Média de Atendimentos de Urgência Odontológica por Habitante e Taxa de Incidência de Alterações na Mucosa oral, 40,1\% ( $\mathrm{n}=55)$ dos profissionais sabiam o local correto para o registro das informações.

Tabela 2. Registro das respostas dos Cirurgiões-Dentistas da Atenção Básica do município quanto à forma de registro dos procedimentos, Fortaleza, Ceará, 2014

\begin{tabular}{lcrcr}
\hline PROCEDIMENTOS & \multicolumn{2}{c}{$\begin{array}{c}\text { Total de profissionais que } \\
\text { menciona o local correto para } \\
\text { o registro das informações }\end{array}$} & $\begin{array}{c}\text { Total de profissionais que não } \\
\text { menciona o local correto para } \\
\text { o registro das informações }\end{array}$ \\
\cline { 2 - 5 } & $\mathbf{n}$ & $\mathbf{\%}$ & $\mathbf{n}$ & \% \\
\hline Ação coletiva de escovação dental supervisionada & 36 & 26,3 & 101 & 73,7 \\
Primeira consulta odontológica programática & 56 & 40,9 & 81 & 59,1 \\
Instalação de prótese dentária & 30 & 21,9 & 107 & 78,1 \\
Atendimento odontológico à gestante & 55 & 40,1 & 82 & 59,9 \\
Atendimento de urgência odontológico & 55 & 40,1 & 82 & 59,9 \\
na Atenção Básica & 55 & 40,1 & 82 & 59,9 \\
Diagnóstico de alteração da mucosa oral & & & & \\
\hline
\end{tabular}

Fonte: Elaboração própria 
Para que as informações possam contribuir para o desenvolvimento dos modelos de atenção, torna-se necessária sua divulgação entre todos os profissionais e que sejam disponibilizados à população (MOTA; CARVALHO, 1999). Isso pode estar inserido na proposta de EP de cada categoria profissional ligada à ESF ou, até mesmo, em reuniões dos conselhos locais de saúde. Tais momentos são identificados como espaços de troca entre os participantes e poderiam contribuir, sobremaneira, para a discussão e a identificação de estratégias de superação dos problemas na saúde pública.

Almeida e Ferreira (2008) expõem que as informações acerca da regularidade e da frequência dos procedimentos preventivos em saúde são escassas. Parece não haver regularidade alguma no processo de informação em saúde. A partir disso, pode-se mencionar que um dos principais obstáculos à implementação de processos avaliativos em saúde bucal é a baixa qualidade dos sistemas de informações disponíveis (SCOCHI, 1994).

\section{Considerações finais}

Os resultados obtidos nesta pesquisa apresentam algumas informações importantes para o aprimoramento do processo de avaliação junto às EqSB da ESF no município de Fortaleza, Ceará. Pôde-se constatar que os CDs são, de certa forma, experientes no serviço e que grande parte já realizou ou realiza alguma pós-graduação, sendo $60 \%$ dos cursos, aproximadamente, nas áreas de saúde da família, saúde pública e/ou saúde coletiva.

No que concerne aos indicadores de desempenho e monitoramento adotados pelo Ministério da Saúde, a grande maioria dos entrevistados os considera importantes, no entanto, apontam deficiências nos serviços que precisam ser aprimorados pela gestão, tais como: intensificação das políticas de EP junto às EqSB; manutenção regular dos equipamentos e insumos odontológicos; melhoria da estrutura das EqSB, sobretudo nos consultórios odontológicos, equipamentos periféricos e instrumentais; ampliação da cobertura das EqSB nos territórios do município de Fortaleza; e melhorias nas relações de trabalho e no planejamento da gestão para organização da demanda e adesão dos pacientes às consultas programadas.

Com isso, cabe à gestão municipal de Fortaleza buscar, na medida do possível, adotar estratégias que venham a responder, gradativamente, às possíveis deficiências supracitadas, na perspectiva de tornar a gestão cada vez mais resolutiva e para que essas iniciativas possam promover uma melhoria efetiva na qualidade de vida das pessoas, em especial, daqueles que mais necessitam. 


\section{Referências}

ALBUQUERQUE, O. M. R.; ABEGG, C.; RODRIGUES, C. S. Percepção de gestantes do Programa de Saúde da Família em relação a barreiras no atendimento odontológico em Pernambuco, Brasil. Cad. Saúde Pública, Rio de Janeiro, v. 20, n. 3, p. 786-796, 2004.

ALMEIDA, G. C. M.; FERREIRA, M. A. F. Saúde bucal no contexto do Programa Saúde da Família: práticas de prevenção orientadas ao indivíduo e ao coletivo. Cad. Saúde Pública, Rio de Janeiro, v. 24, n. 9, p. 2131-2140, 2008.

ALMEIDA, P. F.; GIOVANELLA, L. Avaliação em Atenção Básica à Saúde no Brasil: mapeamento e análise das pesquisas realizadas e/ou financiadas pelo Ministério da Saúde entre os anos de 2000 e 2006. Cad. Saúde Pública, Rio de Janeiro, v. 24, n. 8, p. 1727-1742, 2008.

ALVES, V. S. Um modelo de educação em saúde para o Programa Saúde da Família: pela integralidade da atenção e reorientação do modelo assistencial. Interface (Botucatu), Botucatu, v. 9, n. 16, p. 36-48, 2005.

BEATTIE, A. The health promotion school: from idea to action. In: SCRIVEN A, ORME J. Health promotion: professional perspectives. Hampshire: MacMillan The Open University; 1996. p. 129-143.

BRASIL. Ministério da Saúde. Secretaria de Atenção à Saúde. Departamento de Atenção Básica. Programa Nacional de Melhoria do Acesso e da Qualidade da Atenção Básica (PMAQ): manual instrutivo. Brasília: Ministério da Saúde, 2012.

CHIARATTO, R. A. A importância do cirurgiãodentista como agente promotor do aleitamento materno. Amamentação Online - Grupo de Apoio ao Aleitamento. 2003. Disponível em: <http://www.aleitamento.com/livraria/bases-cientificas-profissionais. asp>. Acesso em: 03 mar 2013.

FREITAS, C. H. S. M. Dilemas no exercício profissional da Odontologia: a autonomia em questão. Interface (Botucatu), Botucatu, v. 11, n. 21, p. 25-38, 2007.

GOMEZ, I. et al. Is early diagnosis of oral cancer a feasible objective? Who is to blame for diagnostic delay? Oral Dis, Copenhagen, v. 16, n. 4, p. 333-342, 2010.

HAMALAINEN, P. et al. Oral infections as predictors of mortality. Gerodontology, Oxford, v. 22, n. 3, p. 151-157, 2005.

MAEDA, F. H. I.; IMPARATO, J. C. P.; BUSSADORI, S.
K. Atendimento de pacientes gestantes: a importância do conhecimento em saúde bucal dos médicos ginecologistas-obstetras. Revista Gaúcha de Odontologia, Porto Alegre, v. 53, n. 1, p. 59-62, 2005.

MCDOWELL, J. D. An overview of epidemiology and common risk factors for oral squamous cell carcinoma. Otolaryngol Clin North Am., Philadelphia, v. 39, n. 2, p. 277-294.

MIOTTO, M. H. M. B. et al. Avaliação do impacto na qualidade de vida causado por problemas bucais na população adulta e idosa em município da Região Sudeste. Ciência \&t Saúde Coletiva, Rio de Janeiro, v. 17, n. 2, p. 397-406, 2012

MORITA, M. C.; HADDAD, A. E.; ARAÚJO, M. E. Perfil atual e tendências do cirurgião-dentista brasileiro. Maringá: Dental Press International, 2010.

MOTA, E.; CARVALHO, D. M. Sistemas de informação em saúde. In: ROUQUAYOROL, M. Z.; ALMEIDA FILHO N. Epidemiologia e saúde. 5. ed. Rio de Janeiro: Medsi; 1999. p. 505-521.

MOYSÉS, S. J. O futuro da Odontologia, no Brasil e no mundo, sob o ponto de vista da Promoção da Saúde. Rev. Bras. Odontol., São Paulo, v. 16, n. 1, p. 10-13, 2008.

NUTBEAM, D. Promotion health and preventing disease: an international perspective on youth health promotion. $J$ Adolesc Health, New York, v. 20, n. 5, p. 396-402, 1997.

SCOCHI, M. J. Indicadores de qualidade dos registros e da assistência ambulatorial em Maringá, (Estado do Paraná, Brasil), 1991: um exercício de avaliação. Cad Saúde Pública, São Paulo, v. 10, n. 3, p. 356-367, 1994.

SHEIHAM, A. Promoting periodontal health - effective programmaes of education and promotion. Int Dent J., London, v. 33, n. 2, p. 182-187, 1983.

WATT, R. G. From victim blaming to upstream action: tackling the social determinants of oral health inequalities. Community Dent Oral Epidemiol., Copenhagen, v. 35 , n. 1, p. 1-11, 2007

Recebido para publicação em abril de 2014

Versão final em agosto de 2014

Conflito de interesses: inexistente

Suporte financeiro: não houve 